Pacific Journal of Mathematics

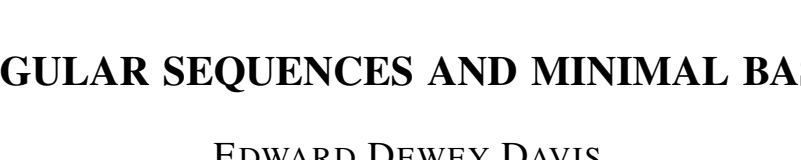




\title{
REGULAR SEQUENCES AND MINIMAL BASES
}

\author{
EDWARD D. DAVIS
}

This note records the results of an effort to understand in simple terms a certain theorem of Lichtenbaum and Schlessinger : Let $I \supset J$ be ideals of noetherian local ring. If $I$ and $I / J$ are generated by regular sequences, then so is $J$. This theorem is closely related to the well known: If $R$ and $R / P$ are regular local rings, then $P$ is generated by part of a regular system of parameters. We investigate the implications of " $I / J$ is generated by a regular sequence" and discover an elementary theorem having both of these results as corollaries.

1. Notation and preliminaries. We consider only commutative rings with $1 \neq 0$ and adopt the convention that local and semilocal rings need not be noetherian. Given a finitely generated ideal (or module) $X$, we denote by $\nu(X)$ the greatest integer $n$ such that $X$ has no basis of fewer than $n$ elements; a basis of cardinality $\nu(X)$ will be called a minimal basis. We denote the following elementary fact as Lemma A: Every basis of a module over a local ring contains a minimal basis. Recall that a regular sequence in a ring $R$ is a finite sequence $\left(x_{1}, \cdots, x_{n}\right)$ of elements of $R$ such that $R x_{1}+\cdots+R x_{n} \neq R$ and $x_{i}$ is not a divisor of zero on $R / R x_{1}+\cdots+R x_{i-1}(1 \leqq i \leqq n)$. If the ideal $X$ is generated by the members of such a sequence, then $\nu(X)=n$, for it is an easy exercise to check that $X / X^{2}$ is a free $(R / X)$-module of rank $n$. We shall have need of another elementary fact which we shall denote by Lemma $B$ : If an ideal of noetherian local ring is generated by a regular sequence, then any permutation of a minimal basis of the ideal is a regular sequence. (A good account of all the elementary facts we need can be found, for example, in Kaplansky's book [2]; specifically, Lemma $\mathrm{A}$ is contained in Theorem 158, Lemma $B$ in Theorem 129, and $\S 3-1$ deals with regular sequences.) We shall be concerned with results of the type $\nu(I)=\nu(I / J)+\nu(J)$. This equation is clearly equivalent to: The union of a minimal basis of $J$ with a set of representatives of a minimal basis of $I / J$ is a minimal basis of $I$.

2. Noetherian local rings. In this section $I \supset J$ are ideals of ideals of a noetherian local ring $R$. The relaxation of the conditions "local" and "noetherian" will be discussed in $\S 3$.

THEOREM. If $I / J$ is generated by a regular sequence, then $\nu(I)=\nu(I / J)+\nu(J)$. 
COROLlaRy 1. (Lichtenbaum and Schlessinger [3, 3.3.4]). If both $I$ and $I / J$ are generated by regular sequences, then so is $J$.

Corollary 2. If $R / J$ is regular, then $J$ is generated by part of a minimal basis of the maximal ideal. (Hence, if $R$ and $R / J$ are regular, then $J$ is generated by part of a regular system of parameters.)

Corollary 2 is well known; its geometric content is: if $p$ is a simple point of a subvariety $W$ of a variety $V$, then "locally at $p$ " $W$ "looks" like the intersection of $V$ with a linear space.

Proofs. The corollaries follow immediately from the theorem; the first by Lemma B, and the second by the well known fact that the maximal ideal of a regular local ring is generated by a regular sequence. To prove the theorem notice that by the hypothesis and Lemma A, $I$ has a minimal basis $\left\{x_{i}, \cdots, x_{n}, y_{1}, \cdots, y_{m}\right\}$, where the $y$ 's lie in $J$ and $x$ 's form a regular sequence $\bmod J$. By passing to $R / R y_{1}+\cdots+R y_{m}$, we may assume that $\nu(I)=\nu(I / J)$ and must prove that $J=0$. We proceed by induction on $n$. For $n=1$, since $x_{1}$ is not a divisor of zero $\bmod J, J=J x_{1}$, whence $J=0$ by Nakayama's Lemma. For $n>1$, an application of the case $n=1$ to the ring $R / R x_{1}+\cdots+R x_{n-1}$ shows that the canonical image of $J$ is 0 ; that is, $R x_{1}+\cdots+R x_{n-1} \supset J$, whence $J=0$ by the induction hypothesis.

REMARK 1. The theorem also admits a proof by the methods of Lichtenbaum and Schlessinger. The exact sequence of the triple $(R, R / I, R / J)$ applied to the $(R / J)$-module $R / J$ together with the fact that $I / J$ is generated by a regular sequence gives the exactness of $0 \rightarrow J / I J \rightarrow I / I^{2} \rightarrow I / J+I^{2} \rightarrow 0[3,3.2 .1$ and proof of 3.3.4]. Since $\mathrm{I} / J+I^{2}$ is $(R / I)$-free, the sequence splits, whence by "local", $\nu\left(I / I^{2}\right)=$ $\nu(J / I J)+\nu\left(I / J+I^{2}\right)$. The theorem follows since $\nu\left(I / I^{2}\right)=\nu(I)$, $\nu(J / I J)=\nu(J)$, and $\nu\left(I / J+I^{2}\right)=\nu(I / J)$ by Nakayama's Lemma.

REMARK 2. Let $A \supset B$ be finitely generated $R$-modules, and let $S$ denote the sequence $0 \rightarrow B \rightarrow A \rightarrow A / B \rightarrow 0$. Observe that for any ideal $N, S \otimes R / N$ is exact if, and only if, $B \cap N A=N B$. With this in mind one routinely abstracts the argument of the last two sentences of the preceding paragraph to prove the equivalence of:

(1) $\nu(A)=\nu(A / B)+\nu(B)$.

(2) For $M$ the maximal ideal of $R, S \otimes R / M$ is exact (if, and only if, $B \cap M A=M B$ ).

(3) There exists an ideal $N$ such that $S \otimes R / N$ is exact (if, and only if, $B \cap N A=N B)$ and $A / B \otimes R / N$ is $(R / N)$-free. 
(4) There exists an ideal $N$ such that $S \otimes R / N$ is split exact. Generalization of the theorem beyond the realm of ideals generated by regular sequences is probably best studied through (4); that is, one could look for classes of pairs $A \supset B$ for which reasonable candidates for $N$ would present themselves. In general (3) is probably not likely to be an improvement over (2) since one cannot reasonably expect the freeness of $A / B \otimes R / N$ for many $N$ 's.

REMARK 3. In view of the previous remarks we see that the Lichtenbaum-schlessinger proof of the theorem amounts to showing that $J \cap I^{2}=I J$. Following the referee's suggestion to examine the appropriate Koszul complex we see where this point lies buried in our proof. The choice of the $x$ 's guarantees that the complex obtained by tensoring with $R / J$ the Koszul complex they generate has vanishing homology in dimension 1; and the long exact sequence of "Koszul homology" associated to $0 \rightarrow J \rightarrow R \rightarrow R / J \rightarrow 0$ then gives the exactness of $0 \rightarrow J / K J \rightarrow R / K \rightarrow R / I \rightarrow 0$, where $K=R x_{1}+\cdots+R x_{n}$ (for details see, e.g. [1, 1.2 and 1.5]). Thus $J \cap K=K J$ from which $J \cap I^{2}=I J$ immediately follows. (Actually our proof reduced to the case $I=K$ and showed $J=I J$ which this argument also shows.)

3. On extending the results of $\S 2$. Graded rings. We consider only graded rings $R$ such that $R_{0}$ is a field and $R_{i}=0$ for $i<0$. As a rule theorems about local rings translate into theorems in the graded situation, and that is true of the results of $\S 2$. The translations are accomplished via the graded versions of Lemmas $A$ and $B$ which are valid for homogeneous ideals and bases even without the noetherian hypothesis in Lemma B. The proof of the theorem works in the graded case without any assumption other than that $I$ and $J$ be homogeneous ideals; and it follows that the Lichtenbaum-Schlessinger result holds for homogeneous ideals in graded rings. The translation of Corollary 2 requires that $R=R\left[R_{1}\right]$ and that $R_{1}$ be of finite $R_{0}$-dimension (i.e., $R$ is the quotient of a polynomial ring by a homogeneous ideal); whence: If $J$ is a homogeneous ideal such that $R / J$ is isomorphic (as an abstract ring) to a polynomial ring, then $J$ is generated by a subspace of $R_{1}$.

Local rings. Our theorem is valid for arbitrary local rings provided that $J$ is finitely generated, for that is the extent to which the proof requires "noetherian". That some vestige of "noetherian" must remain is clear-consider any valuation ring of Krull dimension greater than 1. Remark 2 is clearly valid for arbitrary local rings. Concerning Remark 3 , observe that " $0 \rightarrow J / I J \rightarrow I / I^{2} \rightarrow I / J+I^{2} \rightarrow 0$ is split exact" is a formal consequence of the vanishing of the appropriate "Koszul 
homology" in dimension 1; no other hypothesis is needed (not even "local") since that condition implies the $(R / I)$-freeness of $I / J+I^{2}$ as well as the exactness of $0 \rightarrow J / K J \rightarrow R / I \rightarrow R / K \rightarrow 0$. (Of course this condition on the homology is equivalent in the noetherian local case to "the $x$ 's form a regular sequence $\bmod J$ " [1, 2.8].) Stated for modules over local rings our theorem becomes: If $A \supset B$ are finitely generated submodules of $C$ such that $A=B+C x_{1}+\cdots+C x_{n}$, where the $x$ 's form a $(C / B)$-regular sequence, then $\nu(A)=\nu(A / B)+\nu(B)$. The proof given in $\S 2$ works here-as does the proof of the generalization assuming only the vanishing in dimension 1 of the homology of the complex obtained by tensoring with $C / B$ the Koszul complex generated by the $x$ 's. Recalling Remark 2 and letting $N=$ $\mathrm{R} x_{1}+\cdots+R x_{n}$, one can observe that here we have a case in which $S \otimes R / N$ is split exact with $A / B \otimes R / N$ not necessarily $(R / N)$-free.

Semilocal rings. An easy application of the Chinese Remainder Theorem shows that for semilocal $R, \nu(X)=\max \left\{\nu\left(X \otimes R_{M}\right\}\right.$, where $M$ runs over the maximal ideals of $R$. With this fact and the local version of the theorem one readily proves that our theorem holds for arbitrary semilocal rings provided that $I$ is a radical ideal (i. e., contained in every maximal ideal). Then because Lemma B holds for radical ideals in noetherian rings, it follows that the LichtenbaumSchlessinger result is valid for radical ideals in noetherian semilocal rings.

\section{REFERENCES}

1. M. Auslander and D. A. Bachsbaum, Codimension and multiplicity, Annals of Math., 68 (1958), 625-657.

2. I. Kaplansky, Commutative Rings, Boston: Allyn and Bacon, 1970.

3. S. Lichtenbaum and M. Schlessinger. The cotangent complex of a morphism, Trans. Amer. Math. Soc., 128 (1967), 41-70.

Received March 16, 1970.

State University of New York, Albany 


\section{PACIFIC JOURNAL OF MATHEMATICS}

\section{EDITORS}

H. SAMELson

Stanford University

Stanford, California 94305

R. R. PHelPS

University of Washington

Seattle, Washington 98105
J. Dugundu

Department of Mathematics

University of Southern Californle

Los Angeles, California 9.0007

RICHARD ARENS

University of California

Los Angeles, California 9.0024

\section{ASSOCIATE EDITORS}
E. F. BECKENBACH
B. H. NeumanN
F. WOLE
K. Yoshida

\section{SUPPORTING INSTITUTIONS}

UNIVERSITY OF BRITISH COLUMBIA

CALIFORNIA INSTITUTE OF TECHNOLOGY

UNIVERSITY OF CALIFORNIA

MONTANA STATE UNIVERSITY

UNIVERSITY OF NEVADA

NEW MEXICO STATE UNIVERSITY

OREGON STATE UNIVERSITY

UNIVERSITY OF OREGON

OSAKA UNIVERSITY

UNIVERSITY OF SOUTHERN CALIFORNIA

\author{
STANFORD UNIVERSITY \\ UNIVERSITY OF TOKYO \\ UNIVERSITY OF UTAH \\ WASHINGTON STATE UNIVERSITY \\ UNIVERSITY OF WASHINGTON \\ AMERICAN MATHEMATICAL SOCIETY \\ CHEVRON RESEARCH CORPORATION \\ NAVAL WEAPONS CENTER
}

The Supporting Institutions listed above contribute to the cost of publication of this Journal, but they are not owners or publishers and have no responsibility for its content or policies.

Mathematical papers intended for publication in the Pacific Journal of Mathematics should be in typed form or offset-reproduced, (not dittoed), double spaced with large margins. Underline Greek letters in red, German in green, and script in blue. The first paragraph or two must be capable of being used separately as a synopsis of the entire paper. The editorial "we" must not be used in the synopsis, and items of the bibliography should not be cited there unless absolutely necessary, in which case they must be identified by author and Journal, rather than by item number. Manuscripts, in duplicate if possible, may be sent to any one of the four editors. Please classify according to the scheme of Math. Rev. Index to Vol. 39. All other communications to the editors should be addressed to the managing editor, Richard Arens, University of California, Los Angeles, California, 90024.

50 reprints are provided free for each article; additional copies may be obtained at cost in multiples of 50 .

The Pacific Journal of Mathematics is published monthly. Effective with Volume 16 the price per volume (3 numbers) is $\$ 8.00$; single issues, $\$ 3.00$. Special price for current issues to individual faculty members of supporting institutions and to individual members of the American Mathematical Society: $\$ 4.00$ per volume; single issues $\$ 1.50$. Back numbers are available.

Subscriptions, orders for back numbers, and changes of address should be sent to Pacific Journal of Mathematics, 103 Highland Boulevard, Berkeley, California, 94708.

PUBLISHED BY PACIFIC JOURNAL OF MATHEMATICS, A NON-PROFIT CORPORATION

Printed at Kokusai Bunken Insatsusha (International Academic Printing Co., Ltd.), 7-17, Fujimi 2-chome, Chiyoda-ku, Tokyo, Japan. 


\section{Pacific Journal of Mathematics}

\section{Vol. 36, No. 2 December, 1971}

George E. Andrews, On a partition problem of H. L. Alder ............ 279

Thomas Craig Brown, An interesting combinatorial method in the theory of locally finite semigroups .......................... 285

Yuen-Kwok Chan, A constructive proof of Sard's theorem ............. 291

Charles Vernon Coffman, Spectral theory of monotone Hammerstein

operators...................................... 303

Edward Dewey Davis, Regular sequences and minimal bases .......... 323

Israel (Yitzchak) Nathan Herstein and Lance W. Small, Regular elements in

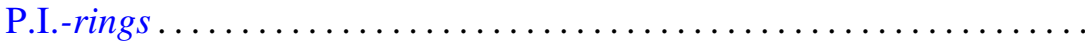

Marcel Herzog, Intersections of nilpotent Hall subgroups ..............

W. N. Hudson, Volterra transformations of the Wiener measure on the space

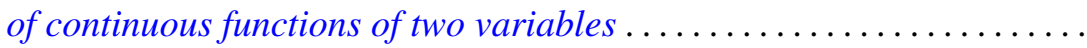

J. H. V. Hunt, An n-arc theorem for Peano spaces ................ 351

Arnold Joseph Insel, A decomposition theorem for topological group

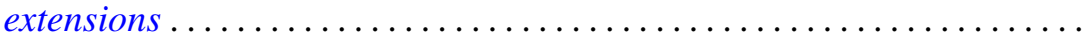

Caulton Lee Irwin, Inverting operators for singular boundary value

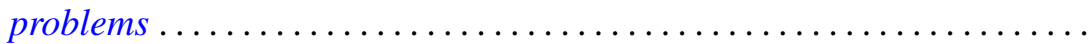

Abraham A. Klein, Matrix rings of finite degree of nilpotency ............ 387

Wei-Eihn Kuan, On the hyperplane section through a rational point of an algebraic variety...

John Hathway Lindsey, II, On a six-dimensional projective representation of $\mathrm{PSU}_{4}(3)$

Jorge Martinez, Approximation by archimedean lattice cones ...

J. F. McClendon, On stable fiber space obstructions .........

Mitsuru Nakai and Leo Sario, Behavior of Green lines at the Kuramochi boundary of a Riemann surface ....................

Donald Steven Passman, Linear identities in group rings. I. .

Donald Steven Passman, Linear identities in group rings. II ...

David S. Promislow, The Kakutani theorem for tensor products of

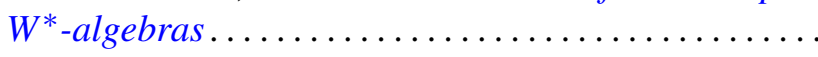

Richard Lewis Roth, On the conjugating representation of a finite group

Bert Alan Taylor, On weighted polynomial approximation of entire functions...

William Charles Waterhouse, Divisor classes in pseudo Galois

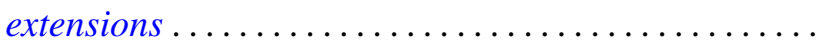

Chi Song Wong, Subadditive functions ...

Ta-Sun $\mathrm{Wu}$, A note on the minimality of certain bitransformation groups 